\title{
Research on calibration technique UHF partial discharge detector in uniformly electric field
}

\author{
Zhou Wei, A , Zhang Jun ${ }^{1, B}$, Liang Xing ${ }^{2, C}$, Chen Bin ${ }^{3, D}$, \\ Gong Jin Long ${ }^{4}$ E \\ ${ }^{1}$ China Electric Power Research Institute, China \\ ${ }^{2}$ Chong Qing Electric Power Company Electric Power Research Institute, China \\ ${ }^{3}$ Tian Jin Electric Power Company Electric Power Research Institute, China \\ ${ }^{4}$ Zhe Jiang Electric Power Company Electric Power Research Institute, China) \\ azhouweiwinston@163.com , ${ }^{\mathrm{b} z h a n g j u n 3 @ e p r i . s g c c . c o m . c n},{ }^{\mathrm{c} l i a n g x i n g @ c q . s g c c . c o m . c n}$, \\ bin.chen1@tj.sgcc.com.cn, ${ }^{\mathrm{e}}$ gong jinlong@zj.sgcc.com.cn
}

\begin{abstract}
Keywords: electric field intensity; calibration; UHF; Ultra-high frequency partial discharge.
Abstract. In order to achieve accurate measurements of UHF partial discharge detector of key parameters, we developed a calibration device, which can be used for UHF partial discharge detector calibration. This device belongs to the microwave field strength measurement field, we have established a measurement system based on standard field method (computable field). RF signal generator as a calibration signal source, the RF power amplifier module for small signal amplification, calibration system also includes a coaxial connector, GTEM cell, field probes, measurement module, main control module. We design and optimize the GTEM cell of field uniformity, and constructed a test area high accuracy, and within $300 \mathrm{MHz} \sim 1.5 \mathrm{GHz}$ frequency range tested. Calibration system with wideband, high accuracy and other characteristics, and solves the partial discharge UHF detector traceability issues, to meet the demand for UHF partial discharge detection instrument calibration.
\end{abstract}

\section{Introduction}

The physical nature of the partial discharge UHF signal is transient ultra-high frequency electromagnetic pulse, the physical nature of UHF partial discharge detection device is a measure of the electric field strength, and this lack of effective traceability of measurement instruments and calibration systems, a reflection of the uncertainty of the calibration system the detection device calibration results trustworthiness, so we need to design a calibration system, and carry out research of the main parameters of traceability methods. Demand for calibration UHF partial discharge detection device, an urgent need for the establishment of a set of reference conditions based on standard field method (computable field) calibration system, in order to solve UHF Partial Discharge Detector charged traceability issues, and meet UHF partial discharge detection device calibration requirements.

About Partial Discharge UHF detector test technology has a variety of research, CIGRE TF15/33.03.05 recommended a discharge on GIS entities analog signal and calibration depends on the amount of charge of checking methods. This method is recommended CIGRE site adaptability is not high, very high requirements for the preliminary work, heavy workload; and signal injection point selected site difficult, difficult to ensure the strength of the signal injection; only qualitative analysis, quantitative analysis is difficult to accurately; amount value can't be traceable. Calibration device is only capable of UHF PD sensors calibrate the instrument, but can't put the entire measurement system calibration UHF Bureau. Calibration device is too dependent on the reference sensor, the sensor type, size, orientation, connectors and other aspects will have a huge impact on the measured reference amount, thus introducing errors can't be ignored.

Present domestic and international for calibration UHF partial discharge monitoring system has not been uniform and consistent conclusions accredited calibration means. UHF partial discharge detection accuracy measurement data live, and reliability can't be properly evaluated, leading to risks of UHF partial discharge monitoring, and thus the formation of the power system security risks. 
Therefore, the development of an environment in the laboratory charged UHF partial discharge measurement standard detector device has become an important and urgent technical problems that need to be overcome, its data reliability evaluation method is for further study.

\section{Constitute Measurement Standards Apparatus}

UHF partial discharge detector is used to measure the ultra-high frequency (UHF) electromagnetic wave signal of special equipment. UHF partial discharge detector standard means in essence is a set of radio metering devices, the main parameters which measures the electric field strength.Mainly traceable standard metering device UHF partial discharge detector is $300 \mathrm{MHz} \sim 3 \mathrm{GHz}$ field strength. When UHF partial discharge detection instrument calibration of field strength, need to build a standard electric field, and a certain GTEM cell design to meet its field uniformity requirements, and UHF partial discharge detector sensor into a specific field area internal calibration. IEEE Std 1309-2005 released on standard field probes and sensors recommended the following three kinds of calibration methods. Research field calibration method based on the IEEE standard, that is, the sensor will be placed in a calibrated reference field, in the frequency range of the calibration work involved, the field of the reference field intensity can be calculated by their geometric size, shape, after combining the input parameters measured obtained. Standard field method that is to build a traceable standard field, the field intensity and its spatial distribution can be accurately calculated by the presence of a region within the field strength to meet certain requirements and relatively uniform, according to the standard field method related module design .

UHF partial discharge detector operating frequency band of $300 \mathrm{MHz} \sim 3 \mathrm{GHz}$, to be able to accurately measure the electromagnetic wave signal propagating along the space, not only required to ensure the propagation path UHF electromagnetic signals correctly, and requires the propagation path attenuation control, need to adopt "electromagnetic shielding module "masking its directivity. Electromagnetic shielding modules using a distributed non-inductive resistor and absorbing materials were improved to match the low frequency and high frequency, when its beginning excitation power fed into the inner electromagnetic shielding module to establish a uniform line transverse electromagnetic wave, which is free electromagnetic properties of the same far-field region of space, and the device under test or sample "drowned" in the electromagnetic waves, it is possible to simulate the electromagnetic field of the free air environment.

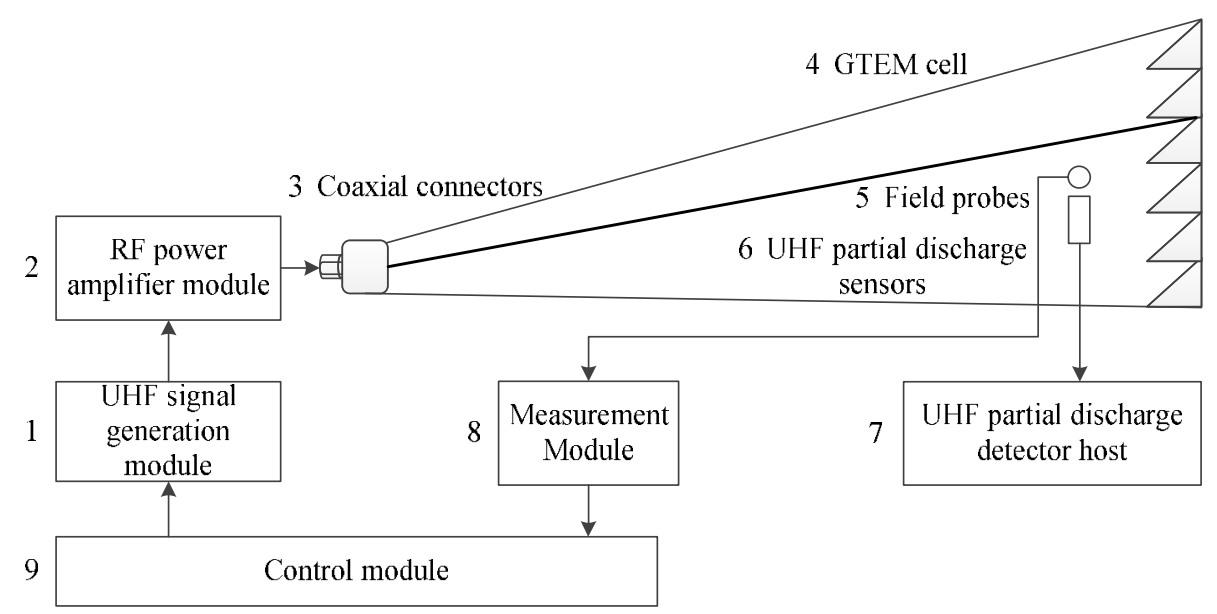

Figure. 1 UHF partial discharge detector calibration system block diagram

Electromagnetic shielding module and RF power input chamber following a fixed relationship. Let the input power is $P$, the core module electromagnetic shield the vertical distance from the base plate $h$, the field strength:

$$
\mathrm{E}=\sqrt{(50 \mathrm{P})} / \mathrm{h}
$$


If we consider the difference between the measured value and the theoretical value, and the formula should be multiplied by a correction coefficient $\mathrm{k}$, so the actual electric field strength:

$$
\mathrm{E}=\mathrm{k} \sqrt{(50 \mathrm{P})} / \mathrm{h}
$$

From the above equation, the strong can be calculated and controlled by calculating its field. PD excited $300 \mathrm{MHz} \sim 3 \mathrm{GHz}$ electromagnetic wave (microwave), live and on-line UHF PD tester is actually measured microwave field strength through the sensor, and can be field-strength values converted voltage line segment, after subsequent signal processing analysis and judgment PD situation. Thus realizing the UHF PD charged detector calibration.

According to the measured properties, UHF PD measurement standard instrument contains the following sections: UHF signal generator module, power amplifier module, coaxial connectors, electromagnetic shielding module (standard field), field probes, measurement module: Module source part generated by UHF signal, power amplifier modules, coaxial connector composition. For generating a frequency $300 \mathrm{MHz} \sim 3 \mathrm{GHz}$ and a maximum output power of $+18 \mathrm{dBm}$ signal in the UHF power amplifier after amplification can generate an electric field is not less than $10 \mathrm{~V} / \mathrm{m}$ in the corresponding field of the environment, which should have a higher coaxial connector the frequency characteristics. Environmental standards field is an electromagnetic shielding module one small area. When the UHF electromagnetic energy fed into the electromagnetic shielding module, a uniform traveling wave electromagnetic field in a specific area of the module can be established, and this standard field area can be calibrated and traceable. Measurement module consists of field probes and measurement modules. The probe volume is smaller, the resolution is higher, the interference of the field is smaller, the probe or antenna into the field of environmental standards, can probe field amplitude of the electric field distribution measurement module output to be measured.

\section{Optimized Design of GTEM}

In order to establish a standard electromagnetic field environment, the field intensity and its spatial distribution can be accurately calculated, and the electric field more uniform. So using GTEM cell provides a standard market environment. The standard field probes and UHF PD sensors simultaneously into a standard field measurements. Electronic shielding module is tapered coaxial structure. In the core around excited transverse electromagnetic wave impedance 377 ohms. Can provide very good field homogeneity and reproducibility field in a given workspace area is also ideal for field calibration.

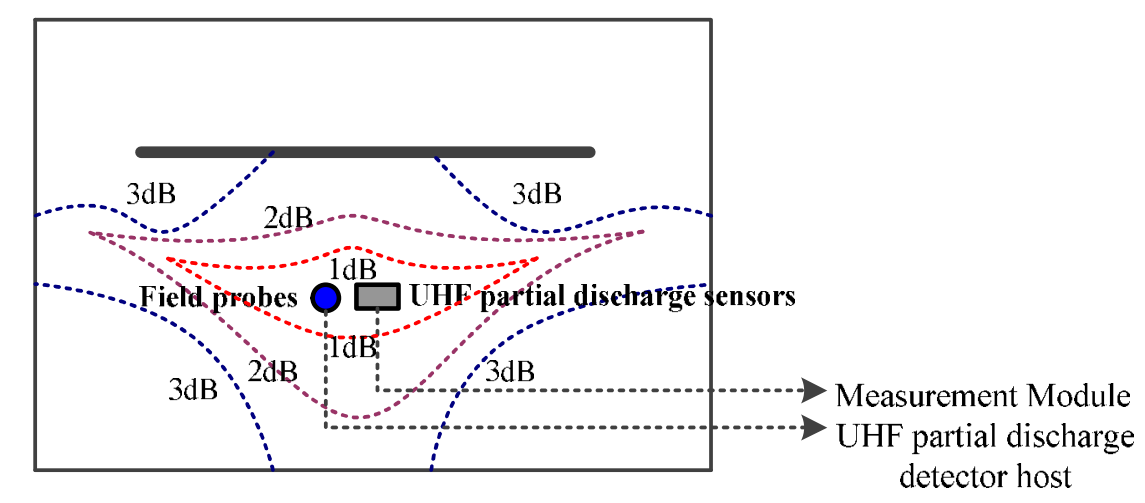

Figure. 2 GTEM cell cross-sectional field distribution

For frequency domain measurements, the choice of sweep signal source, power amplifier, electric field shielding module load. Adjusting the sweep signal source output signal amplitude and amplifier gain, the monitoring is fed to a small indoor power or voltage magnitude, through the measured field distribution curve. After the measured field at the location of the sensor powerful small compared with the magnitude of the output of the sensor response, that is, the transmission coefficient on the 
frequency of transmission coefficient were tested at different frequencies, can be drawn amplitude-frequency sensor response curves, determining $-1 \mathrm{~dB}$ bandwidth. In GTEM cell interior $1 \mathrm{~dB}$ region, its uniform electric field intensity curve shown in Figure 2.

\section{Electric field intensity measurement}

Field probes and UHF PD sensors are "high-frequency microwave field strength metering" category. Microwave field strength measurement of electromagnetic radiation field is divided into near-field and far field, UHF partial discharge test instrument is generally near field of the electric field strength in the near field, low voltage, high current for field source (such as a local UHF discharge), an electric field is much stronger than the magnetic field. Electromagnetic field strength near field changes rapidly with distance, the degree of unevenness in the space is relatively large, in view of this problem, each time you use this UHF PD measurement standards are a response field probe calibration.

Signal Level Meter (interference field strength meter) field test field strength values:

$$
E(\mathrm{~dB} \mu \mathrm{V} / \mathrm{m})=K(\mathrm{~dB})+V \mathrm{r}(\mathrm{dB} \mu \mathrm{V}) / L
$$

Formula correction coefficient $K$ is the antenna, which is a function of frequency, field strength meter in the Schedule by Richard. Level Meter readings must add the corresponding $K$ values $V_{\mathrm{r}}$ and cable loss $L$ to get the field strength values. But the recent production of field strength meter antenna attached $K$ correction factor curve values shown already include measuring antenna cable loss $L$ value.

\section{Test Results}

In UHF partial discharge sensors for electric field strength test, the sensors and the electric field probe, the signal source used is stable continuous sine wave, after the power amplifier amplifies the GTEM small indoor generate stable alternating electric field, the use of an electric field strength probe the electric field strength test point for testing, to ensure the region is a stable field test of the premise, then subsequent sensor calibration. GIS external test sensor is a passive sensor, test frequency range of $300 \mathrm{MHz} \sim 1.5 \mathrm{GHz}$, electric field strength measurement range value change is $6.8 \mathrm{dBm}$ to $44.7 \mathrm{dBm}$.

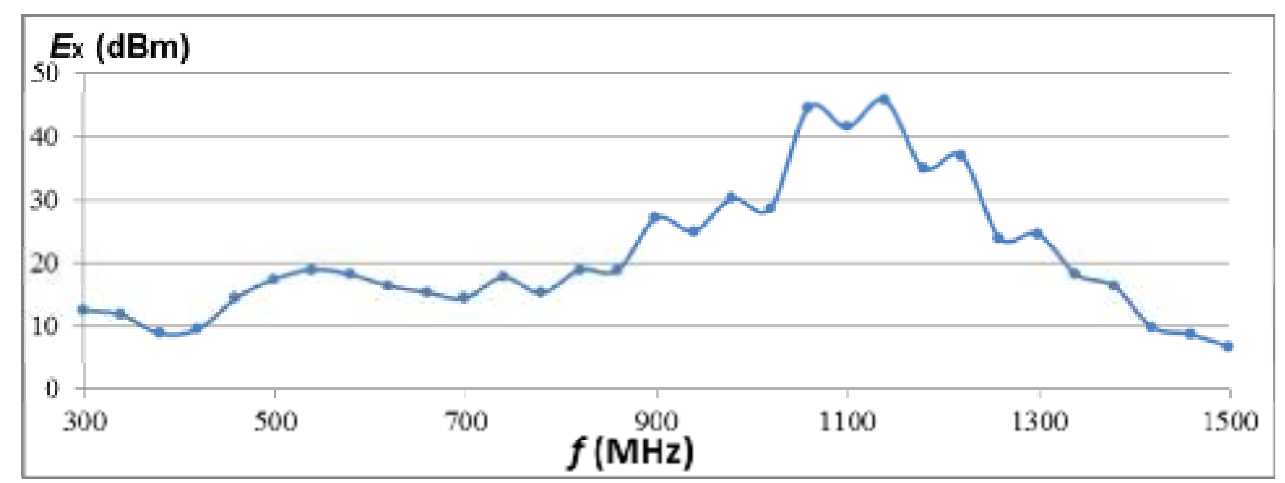

Figure. 3 UHF partial discharge detector test results

\section{Conclusions}

The developed UHF partial discharge detector calibration device has the following characteristics: frequency bandwidth, power, high stability, $1 \mathrm{~dB}$ field area is large. The entire calibration system by the signal generator modules, RF power amplifier module, coaxial connectors, GTEM cell, field probes, measurement module, control module calibration system constituted focusing designed and optimized GTEM cell of field uniformity, through standard field probe to measure the electric field strength values and, using standard field method is designed for UHF partial discharge detector 
calibration system, the establishment of a UHF partial discharge detector measurement standard equipment, the realization of UHF partial discharge sensor field strength values calibration. The system design process of the electric field gradient problem, the electric field strength of the other calibration method and apparatus will be further studied.

\section{References}

[1] IEEE Std 1309-2005 IEEE standard for calibration of electromagnetic field sensors and probes, excluding antennas, from $9 \mathrm{kHz}$ to $40 \mathrm{GHz}$ [S], 2005.

[2] Zhang Mingchao, Wang Jiansheng, Qiu Yuchang. UHF sensors for partial discharge measurement in GIS[J].Power System Technology, 1998, 22(8):42-44.

[3] Wang Feng, Qiu Yuchang. Recent development trend of gas insulated switchgear[J].Power System Technology, 2003, 27(2):54-57.

[4] IEEE Std.1128-1998,IEEE Recommended Practice for RF Evaluation in the Range of $30 \mathrm{MHz}$ $5 \mathrm{GHz}[\mathrm{S}]$.

[5] TANG Shi-ping . Electromagnetic Field Generation Device of Irregular Profile Gigahertz TEM Cell

[6] MA Liang, WU Wei, CHENG Yinhui, et al. Pusled electric field sensor based on original waveform measurement[J]. High Power Laser \& Particle Beams, 2010, 22(11): 2763-2768.

[7] Xue Qianzhong, Ren Liehui, Chen Zhiyu. Correlation between GTEM cell and open area test site in emission measurements[J].Journal of Electronics \& Information Technology, 2003, 25(5):716-720.

[8] Pocai M, Dotto I , Festa D, et al . Improving the performances of a reverberation chamber : a real case $[\mathrm{C}] / / 20$ th International Zurich Symposium on Electromagnetic Compatibility. Switzerland, Zurich: IEEE, 2009:53-56. 